\title{
Shear viscosity of two-state enzyme solutions
}

\author{
Yuto Hosaka and Shigeyuki Komura* \\ Department of Chemistry, Graduate School of Science, \\ Tokyo Metropolitan University, Tokyo 192-0397, Japan \\ David Andelman \\ Raymond and Beverly Sackler School of Physics and Astronomy, \\ Tel Aviv University, Ramat Aviv, Tel Aviv 69978, Israel
}

(Dated: December 31, 2019)

\begin{abstract}
We discuss the shear viscosity of a Newtonian solution of catalytic enzymes and substrate molecules. The enzyme is modeled as a two-state dimer consisting of two spherical domains connected with an elastic spring. The enzymatic conformational dynamics is induced by the substrate binding and such a process is represented by an additional elastic spring. Employing the Boltzmann distribution weighted by the waiting times of enzymatic species in each catalytic cycle, we obtain the shear viscosity of dilute enzyme solutions as a function of substrate concentration and its physical properties. The substrate affinity distinguishes between fast and slow enzymes, and the corresponding viscosity expressions are obtained. Furthermore, we connect the obtained viscosity with the diffusion coefficient of a tracer particle in enzyme solutions.
\end{abstract}

\section{INTRODUCTION}

Molecular enzymes are nanometer-size proteins that catalyze chemical reactions in the presence of substrate molecules. Here substrates are chemical species that react with enzymes and generate product molecules. Catalytic processes that are carried out by molecular enzymes in the cytoplasm and the membrane are essential for cellular metabolism and homeostasis [1]. In the presence of a substrate, enzymes undergo conformational changes in each turnover cycle of the chemical reaction [2]. In order to mimic actual enzymes, these conformational dynamics have been simulated using elastic network models [3 5], and the relationship between conformational dynamics and the chemical reaction stages has been studied recently [6].

One of the long-standing and interesting questions in the field is whether a single enzyme exhibits a motile behavior [7]. Thanks to recent developments of experimental techniques, diffusion phenomena in enzyme solutions have been studied by several groups. Using fluorescence correlation spectroscopy, Muddana et al. [8] reported that diffusion of a single enzyme is enhanced in presence of a substrate. Later on, Riedel et al. [9] showed that the heat released during turnovers also enhances the enzyme diffusion. Illien et al. [10] however, revealed experimentally that not only exothermic enzymes but also endothermic ones contribute to the diffusion enhancement. In the presence of a gradient in substrate concentrations, enzymes exhibit collective motions in the direction of higher or lower concentrations [11, 12]. Moreover, the enhanced diffusion of passive objects in enzymatic solutions have been observed independently [13, 14].

\footnotetext{
* komura@tmu.ac.jp

† andelman@tauex.tau.ac.il
}

To understand these experimental findings, several models have been proposed using equilibrium as well as non-equilibrium approaches. Illien et al. 15] modeled an enzyme consisting of hydrodynamically coupled subunits, and introduced two discrete equilibrium states corresponding to a free enzyme and a substrate-enzyme complex. They showed that diffusion of an enzyme is enhanced due to equilibrium fluctuations 15,16$]$. Within a non-equilibrium framework, Golestanian [17] proposed four possible mechanisms leading to diffusion enhancement by enzymes. They included self-thermophoresis, boost in kinetic energy, stochastic swimming, and collective heating. Mikhailov and Kapral [18, 19] modeled an enzyme as an active force dipole that exerts forces on the surrounding fluid. When such dipoles are immersed in aqueous fluids, hydrodynamic collective effects due to force dipoles can lead to diffusion enhancement [18 20].

In spite of these extensive studies on enzyme diffusion, a recent experimental work pointed out the difficulty of accounting quantitatively for the observed enhanced diffusion within such models as above [21]. Moreover, recent experiments did not observe any change in the diffusion behavior for a specific enzyme that was previously reported to exhibit enhanced diffusion 22, 23]. It was also noticed that the viscosity of enzyme solutions is locally reduced while a specific enzymatic reaction is taking place [7, 24]. However, the effect of enzyme conformational changes on the solution shear viscosity has not been considered theoretically despite its importance.

In this paper, we present an analytical study on the shear viscosity of a dilute enzyme solution under steady shear flow. As a coarse-grained model of catalytic enzymes, we use the two-state dimer model in which conformational changes are induced by substrate binding and product release [18]. Our two-state dimer model consists of two hard spheres representing enzymatic domains, which are connected by a harmonic spring [18, 25, 26] . Assuming that the conformational distribution is given 
by the Boltzmann distribution function, weighted by the waiting time of an enzyme, we obtain analytically the shear viscosity of a two-state dimer solution as a function of the substrate concentration. As a result of the competition between the energy difference of the enzyme two internal states and the substrate concentration, we find that the enzyme solution viscosity exhibits a nonmonotonic behavior that depends on the physical properties of the binding substrates. We shall also connect the obtained viscosity with the diffusion coefficient of a tracer particle in enzyme solutions.

The outline of our manuscript is the following. In Sec. III we review the derivation of the shear viscosity of dimer solutions originally used to describe polymer solutions. In Sec. III we discuss the shear viscosity of a twostate dimer solution that represents enzyme solutions. We first introduce the two-state dimer model and discuss the conformational distribution function of dimers. Analytical results for the shear viscosity due to dimers and its limiting expressions are presented. Finally, some discussions and a summary are given in Sec. IV

\section{VISCOSITY OF DIMER SOLUTIONS}

\section{A. Shear viscosity}

We consider a dilute solution of dimers under steady shear flow as schematically depicted in Fig. 1. Here the solvent viscosity is $\eta_{\mathrm{s}}$ and each dimer is composed of two rigid spheres of radius $a$, which are connected by an elastic spring. The positions of two spheres are denoted by the three-dimensional vectors $\mathbf{r}_{1}$ and $\mathbf{r}_{2}$. Then, the force acting between the two spheres within the dimer is given by

$$
f_{\alpha}=-\frac{\partial U(r)}{\partial r_{\alpha}}
$$

where $U(r)$ is the elastic potential energy, $r=|\mathbf{r}|=$ $\left|\mathbf{r}_{2}-\mathbf{r}_{1}\right|$ is the distance between the two spheres, and $r_{\alpha}$ is the $\alpha$-component of the vector $\mathbf{r}=\left(r_{x}, r_{y}, r_{z}\right)$.

In the presence of potential forces, the equation of motion of an overdamped dimer can be written as [27, 28]

$$
\frac{\partial r_{\alpha}}{\partial t}=\frac{2}{\zeta} f_{\alpha}-\frac{2 k_{\mathrm{B}} T}{\zeta} \frac{\partial \ln \psi}{\partial r_{\alpha}}+d_{\alpha \beta} r_{\beta}
$$

where $\zeta$ is the friction coefficient of the sphere, $k_{\mathrm{B}}$ is Boltzmann constant, $T$ is the temperature, $\psi(\mathbf{r}, t)$ is the time-dependent configurational distribution of a dimer, and the velocity gradient tensor is given by

$$
d_{\alpha \beta}=\frac{\partial v_{\alpha}}{\partial r_{\beta}} .
$$

Notice that $v_{\alpha}$ is the $\alpha$-component of the velocity $\mathbf{v}=$ $\left(v_{x}, v_{y}, v_{z}\right)$. Throughout this work, we assume summation over repeated indices. The second and third terms

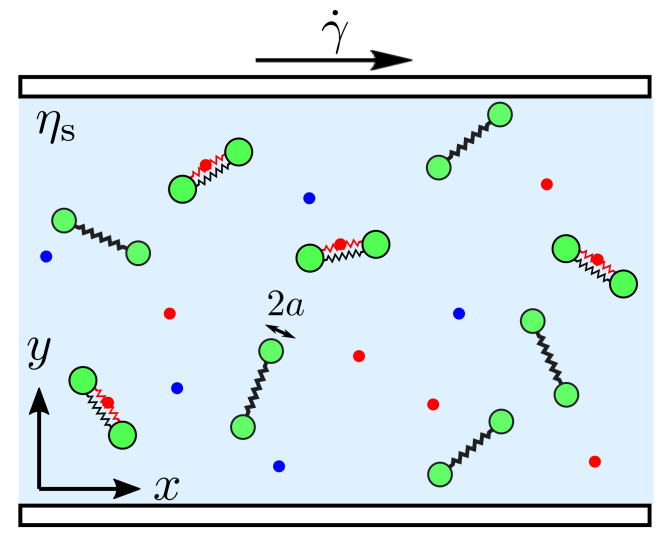

FIG. 1. (Color online) A dilute solution of two-state dimers under steady shear flow with shear rate $\dot{\gamma}$. Dimers consist of two green spheres of radius $a$ connected with an elastic spring, and immersed in a Newtonian fluid having viscosity $\eta_{\mathrm{s}}$. The enzymatic reaction, in which a dimer, a substrate (red circle) and a product (blue circle) participated, is explained in Fig.2

on the right-hand side of Eq. (2) represent the velocity due to thermal motion of the solvent and that imposed by the flow field, respectively.

Such models of dimers have been used extensively to model polymer solutions. For polymer solutions, the stress tensor due to the presence of dimers is given [27, 28]

$$
\sigma_{\alpha \beta}=n\left\langle r_{\alpha} f_{\beta}\right\rangle,
$$

where $n$ is the number density (per unit volume) of dimers, and $\langle\cdots\rangle$ denotes the thermal average over all dimer configurations. To calculate the statistical average in Eq. (4), we introduce the following Fokker-Planck equation for the conformational distribution $\psi(\mathbf{r}, t)$

$$
\frac{\partial \psi}{\partial t}=-\frac{\partial}{\partial r_{\alpha}}\left(\frac{2}{\zeta} f_{\alpha} \psi-\frac{2 k_{\mathrm{B}} T}{\zeta} \frac{\partial \psi}{\partial r_{\alpha}}+d_{\alpha \beta} r_{\beta} \psi\right)
$$

In the above, the continuity equation

$$
\frac{\partial \psi}{\partial t}=-\nabla \cdot\left(\frac{\partial \mathbf{r}}{\partial t} \psi\right)
$$

where $\boldsymbol{\nabla}=\left(\partial r_{x}, \partial r_{y}, \partial r_{z}\right)$ and Eq. (2) have been used. From the time evolution of $\left\langle r_{\alpha} r_{\beta}\right\rangle$ in a steady state, the stress tensor in Eq. (44) can be written as [27, 28]

$$
\sigma_{\alpha \beta}=n k_{\mathrm{B}} T \delta_{\alpha \beta}+\frac{n \zeta}{4}\left[d_{\alpha \gamma}\left\langle r_{\beta} r_{\gamma}\right\rangle+d_{\beta \gamma}\left\langle r_{\alpha} r_{\gamma}\right\rangle\right] .
$$

For simple shear flow whose velocity components are given by $v_{x}=\dot{\gamma} r_{y}, v_{y}=v_{z}=0$, where $\dot{\gamma}$ is the shear rate (see Fig. 1), the viscosity due to dimers has a simple form

$$
\eta=\frac{\sigma_{x y}}{\dot{\gamma}}=\frac{n \zeta}{4}\left\langle r_{y}^{2}\right\rangle
$$

In order to calculate the average $\left\langle r_{y}^{2}\right\rangle$, we need to specify the conformational distribution function $\psi(\mathbf{r})$. 


\section{B. Fraenkel dimer model}

Let us first discuss a dimer consisting of two spheres that are connected by a harmonic spring having an elastic constant $K_{0}$, and a natural length $\ell_{0}$. Its potential energy is then given by

$$
U_{0}(r)=\frac{K_{0}}{2}\left(r-\ell_{0}\right)^{2} .
$$

This is the "Fraenkel dimer model" [29], and is different than other polymer dynamic models, such as the Hookean dimer model. For Fraenkel dimers, the conformational distribution function, $\psi_{0}$, is given by

$$
\psi_{0}(r)=C \exp \left[-\frac{K_{0}}{2 k_{\mathrm{B}} T}\left(r-\ell_{0}\right)^{2}\right],
$$

where $C$ is the normalization constant. Here, we assume that the characteristic relaxation time of a dimer is much smaller than that of a shear flow, i.e., $\zeta \ell_{0}^{2} /\left(k_{\mathrm{B}} T\right) \dot{\gamma} \ll 1$. The physical meaning of this condition will be separately explained in Sec. IV.

Although the shear viscosity of the Fraenkel dimer model was discussed in Ref. [30], its explicit expression was not derived. By calculating $\left\langle r_{y}^{2}\right\rangle$ in Eq. (8) using Eq. (10), we obtain the shear viscosity for a Fraenkel dimer solution $\eta_{0}$ as

$$
\begin{aligned}
& \frac{\eta_{0}(\epsilon)}{G \tau}=\frac{2 \epsilon}{3} \\
& \times \frac{2 \epsilon(5+2 \epsilon) e^{-\epsilon}+\sqrt{\pi \epsilon}\left(3+12 \epsilon+4 \epsilon^{2}\right)[1+\operatorname{erf}(\sqrt{\epsilon})]}{4 \epsilon^{2} e^{-\epsilon}+2 \sqrt{\pi \epsilon}\left(\epsilon+2 \epsilon^{2}\right)[1+\operatorname{erf}(\sqrt{\epsilon})]}
\end{aligned}
$$

where $\epsilon=K_{0} \ell_{0}^{2} /\left(2 k_{\mathrm{B}} T\right)$ is the dimensionless elastic energy, $G=n k_{\mathrm{B}} T$ is the relaxation modulus, $\tau=\zeta /\left(4 K_{0}\right)$ is the relaxation time, and $\operatorname{erf}(x)=(2 / \sqrt{\pi}) \int_{0}^{x} d t e^{-t^{2}}$ is the error function [31]. Notice that $G \tau$ corresponds to the viscosity of a dimer solution when the natural length of the spring vanishes, i.e., $\epsilon=0$ [28, 30].

The limiting behaviors of $\eta_{0}$ for the Hookean, $\epsilon \ll 1$, and stiff Fraenkel dimers, $\epsilon \gg 1$, are given by [27, 30]

$$
\frac{\eta_{0}(\epsilon)}{G \tau}= \begin{cases}1+\frac{4}{3} \sqrt{\frac{\epsilon}{\pi}} & \epsilon \ll 1, \\ \frac{2}{3} \epsilon & \epsilon \gg 1 .\end{cases}
$$

For $\epsilon \ll 1$, the viscosity is almost constant, indicating that thermal energy dominates over elastic energy. For $\epsilon \gg 1$, on the other hand, the viscosity increases linearly with $\epsilon$.

\section{TWO-STATE DIMER SOLUTIONS}

\section{A. Two-state dimer model}

Catalytic enzymes undergo conformational changes in presence of substrate molecules. To model such situations, we use a previously proposed two-state dimer (a)

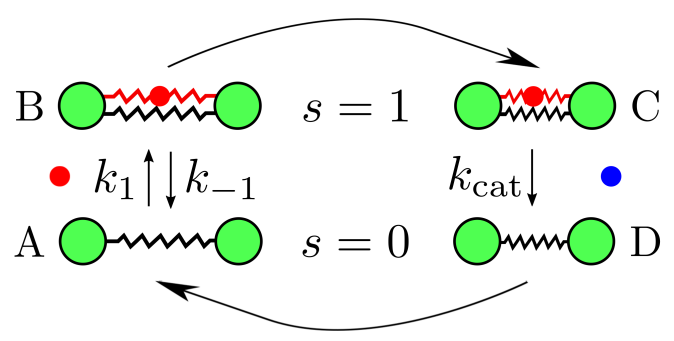

(b)

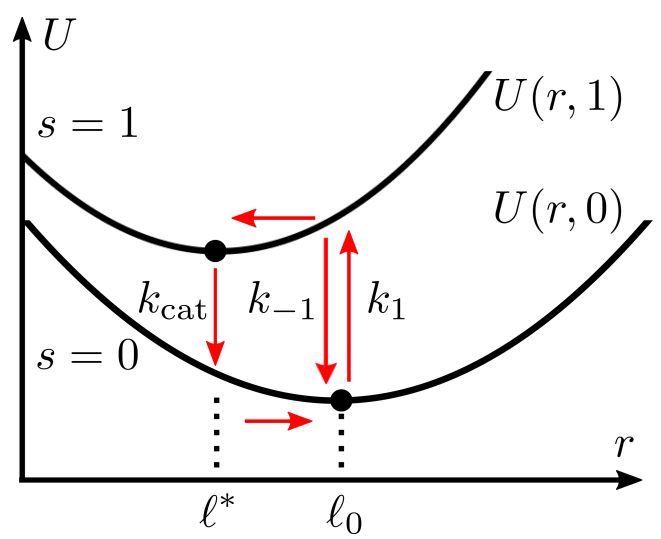

FIG. 2. (Color online) (a) The enzymatic cycle of two-state dimer model. A substrate (red circle) binds to a free enzyme $(s=0)$ with the reaction rate $k_{1}(\mathrm{~A} \rightarrow \mathrm{B})$, while its dissociation also occurs with the reaction rate $k_{-1}(\mathrm{~B} \rightarrow \mathrm{A})$. Once the substrate-enzyme complex $(s=1)$ is formed, it starts to contract until the equilibrium conformation is attained $(B \rightarrow C)$. Then, the product (blue circle) is irreversibly released with the reaction rate $k_{\text {cat }}$, and the bare enzyme comes back to its initial conformation. (b) The schematic illustration of the energy for a two-state dimer as described by Eq. (13). There are two energy branches $U(r, 0)$ and $U(r, 1)$. The transition between them takes place at $r=\ell_{0}$ and $r=\ell^{*}$, which are the equilibrium values of $U(r, 0)$ and $U(r, 1)$, respectively, as are indicated by black circles. This transition is followed by the downhill relaxational motion along each branch. The forward and reverse transition rates, $(s=0) \rightleftarrows(s=1)$, are given by $k_{1}, k_{-1}$, respectively, and $(s=1) \rightarrow(s=0)$ is given by $k_{\text {cat }}$.

model with a state parameter that can get two values, $s=0$ or 1 [18, 25, 26]. In Fig 2(a), we schematically illustrate an enzymatic cycle that is driven by binding a substrate to an enzyme. In the $s=0$ state, i.e., the state of the dimer with the elastic constant $K_{0}$ and the natural length of the spring $\ell_{0}$, this model corresponds to the Fraenkel dimer model.

When a substrate is supplied to a dimer enzyme whose size is $r=\ell_{0}$, a transition from $s=0$ to $s=1$ occurs with the reaction rate $k_{1}$. At the same time, the reverse reaction, namely, the substrate dissociation process, can occur also when $r=\ell_{0}$ with the reaction rate $k_{-1}$. For the state $s=1$, the substrate adds another intra-dimer interaction, which is modeled as an additional spring, whose elastic constant and natural length are $K_{1}$ and $\ell_{1}$, 
respectively. Then, the dimer relaxes to a new equilibrium conformation having the size $r=\ell^{*}$, as will be explicitly given after Eq. (13). Once the substrate molecule is irreversibly converted to a product molecule with the reaction rate $k_{\text {cat }}$, a transition from $s=1$ to $s=0$ takes place at $r=\ell^{*}$. Finally, the product is released from the enzyme.

Notice that the reaction rates, $k_{1}, k_{-1}$ and $k_{\text {cat }}$ are the bare rate constants that do not depend on the energy difference between any two states. This also holds for the reaction rates in the cascade reactions discussed in Appendix A Moreover, the transition of a dimer occurs only when $r=\ell_{0}$ or $r=\ell^{*}$; hence, the reaction rates $k_{1}, k_{-1}$ and $k_{\text {cat }}$ are simply taken to be constant in our model.

The state-dependent total potential energy of this twostate dimer can be written as

$$
U(r, s)=\frac{K_{0}}{2}\left(r-\ell_{0}\right)^{2}+\frac{s K_{1}}{2}\left(r-\ell_{1}\right)^{2},
$$

which gives the equilibrium length for $s=1$ as $\ell^{*}=$ $\left(K_{0} \ell_{0}+K_{1} \ell_{1}\right) /\left(K_{0}+K_{1}\right)$. In Fig. 2(b), we schematically illustrate the energy of a two-state dimer given by Eq. (13) when $\ell_{0}>\ell_{1}$. Under this condition, the substrate-enzyme complex shrinks as compared to the bare enzyme [18, 25, 26]. In this work, however, we do not require such a condition. In physiological conditions, the sizes of actual substrate-enzyme complexes either decrease $\left(\ell_{0}>\ell_{1}\right)$ or increase $\left(\ell_{1}>\ell_{0}\right)$ upon substrate binding [7]. Hereafter, the subscripts " 0 " and " 1 " denote physical values for the enzyme and the substrate-enzyme complex, respectively.

As represented by the second term in the r.h.s. of Eq. (2), a dimer in our model undergoes conformational fluctuations due to thermal energy. In other words, a free enzyme (or a substrate-enzyme complex) fluctuates around $r=\ell_{0}$ (or $r=\ell^{*}$ ) during turnover cycles. This corresponds to the situation in which enzymes are subject to thermal motion of solvent molecules. Notice, however, that conformational fluctuations between multi-state enzymes [32, 33] are not considered. This is because the original dimer model [18, 25, 26] that we employ follows the simple Michaelis-Menten kinetics [see Eq. (14)] with the advantage that the problem becomes tractable.

\section{B. Conformational distribution function}

The above two-state dimer model describes a chemical equation following the standard Michaelis-Menten reaction [34]:

$$
\mathrm{E}+\mathrm{S} \underset{k_{-1}}{\stackrel{k_{1}}{\rightleftarrows}} \mathrm{ES} \stackrel{k_{\mathrm{cat}}}{\longrightarrow} \mathrm{E}_{*}+\mathrm{P}
$$

This chemical reaction equation describes the enzymatic cycle composed of three states of an enzyme: a free enzyme (E), a substrate-enzyme complex (ES), and a free enzyme after the reaction $\left(\mathrm{E}_{*}\right)$, as depicted in Fig. 2 , Furthermore, S and $\mathrm{P}$ stand for the substrate and product, respectively. When dimers are connected by elastic springs, the time spent during the transition between these chemical states can be characterized by a relaxation time $\tau=\zeta /\left(4 K_{0}\right)$ as introduced after Eq. (11).

For a two-state dimer, we assume that the characteristic relaxation time is much smaller than that of a shear flow, i.e., $\zeta \ell_{0}^{2} /\left(k_{\mathrm{B}} T\right) \dot{\gamma} \ll 1$ as adopted for the Fraenkel dimer model in Sec.II. We further assume that the transition time spent between enzymatic states is much smaller than the waiting time in each of the states, $s=0,1$, i.e., $\tau / W_{s} \ll 1$, where the waiting time $W_{s}$ will be defined later in Eq. (16). This assumption is justified for enzymes such as adenylate kinase having a relatively large waiting time, $\tau / W_{1} \approx 0.1[6]$. For completeness, however, the general case of arbitrary waiting times is discussed in Sec. IV Under these conditions, we can introduce the Boltzmann distribution function that is weighted only by the waiting time in the respective enzymatic states. The validity of this assumption has been confirmed by numerical solutions of the Langevin equation for a single two-state dimer [26].

The distribution function for the two-state dimer model for an enzyme is then given by

$$
\psi_{\mathrm{e}}(r)=\frac{W_{0} e^{-\beta U(r, 0)}+W_{1} e^{-\beta U(r, 1)}}{\int d \mathbf{r}\left[W_{0} e^{-\beta U(r, 0)}+W_{1} e^{-\beta U(r, 1)}\right]},
$$

where $\beta=1 /\left(k_{\mathrm{B}} T\right)$. Here the waiting time in the state $s$ is defined by 35,36 .

$$
W_{s}=\int_{0}^{\infty} d t p_{s}(t)
$$

where $p_{s}(t)$ is the time-dependent probability distribution function of an enzyme in state $s$, which will be explicitly given in Eq. (18). The case of a cascade reaction containing $N$ substrate-enzyme complexes is discussed in Appendix $\mathrm{A}$ as a generalization, and Eq. (15), hence, corresponds to the case $N=1$.

\section{Waiting times}

Since we consider a dilute solution of two-state dimers, we employ a single enzyme kinetics to obtain the waiting time that an enzyme spends at each catalytic step (see also Appendix (B). The validity of using a single enzyme kinetics for an enzyme solution will be discussed later in this subsection. For two-state dimers, the corresponding kinetic equations are written in terms of the probability functions as 32, 33, 37, 38.

$$
\begin{aligned}
& \frac{d p_{0}}{d t}=k_{-1} p_{1}-k_{1}^{\prime} p_{0}, \\
& \frac{d p_{1}}{d t}=k_{1}^{\prime} p_{0}-\left(k_{-1}+k_{\text {cat }}\right) p_{1}, \\
& \frac{d p_{*}}{d t}=k_{\text {cat }} p_{1} .
\end{aligned}
$$


Here, $p_{0}(t), p_{1}(t)$ and $p_{*}(t)$ are the probability distribution functions for the two-state dimer in one of the two states, $s=0,1$, and the free enzyme after the catalysis $\left(\mathrm{E}_{*}\right)$, respectively. In the above, we have introduced the pseudo first-order rate constant $k_{1}^{\prime}=k_{1} c_{\mathrm{S}}$, where $c_{\mathrm{S}}$ is the time-independent substrate concentration. Such an assumption is justified when $c_{\mathrm{E}} \ll c_{\mathrm{S}}$ is satisfied, where $c_{\mathrm{E}}$ is the enzyme concentration.

By solving the above coupled kinetic equations using the initial conditions, $p_{0}(0)=1$ and $p_{1}(0)=p_{*}(0)=0$, under the normalization condition $p_{0}(t)+p_{1}(t)+p_{*}(t)=$ 1 , the time-dependent probability distributions are obtained [37]

$$
\begin{aligned}
& p_{0}(t)=\frac{1}{2 a}\left[\left(a+b-k_{1}^{\prime}\right) e^{(a-b) t}+\left(a-b+k_{1}^{\prime}\right) e^{-(a+b) t}\right], \\
& p_{1}(t)=\frac{k_{1}^{\prime}}{2 a}\left[e^{(a-b) t}-e^{-(a+b) t}\right] \\
& p_{*}(t)=\frac{k_{1}^{\prime} k_{\text {cat }}}{2 a}\left[\frac{1}{a-b} e^{(a-b) t}+\frac{1}{a+b} e^{-(a+b) t}\right]+1,
\end{aligned}
$$

where

$$
\begin{aligned}
a & =\left[\left(k_{1}^{\prime}+k_{-1}+k_{\text {cat }}\right)^{2} / 4-k_{1}^{\prime} k_{\text {cat }}\right]^{1 / 2}, \\
b & =\left(k_{1}^{\prime}+k_{-1}+k_{\text {cat }}\right) / 2 .
\end{aligned}
$$

Because $a-b<0$ and $a+b>0$, both $p_{0}(t)$ and $p_{1}(t)$ decay exponentially for $t \rightarrow \infty$, and consequently $p_{*} \rightarrow 1$.

Substituting $p_{0}(t)$ and $p_{1}(t)$ of Eq. (18) into Eq. (16), we obtain the waiting times for $s=0$ and 1 as

$$
W_{0}=\frac{k_{-1}+k_{\mathrm{cat}}}{k_{1}^{\prime} k_{\mathrm{cat}}}, \quad W_{1}=\frac{1}{k_{\mathrm{cat}}} .
$$

As a result, the distribution function in Eq. (15) can be written as

$$
\psi_{\mathrm{e}}(r)=\frac{e^{-\beta U(r, 0)}+\nu e^{-\beta U(r, 1)}}{\int d \mathbf{r}\left[e^{-\beta U(r, 0)}+\nu e^{-\beta U(r, 1)}\right]},
$$

where we have introduced the dimensionless parameter $\nu$

$$
\nu=\frac{k_{1}}{k_{-1}+k_{\text {cat }}} c_{\mathrm{S}}=\frac{c_{\mathrm{S}}}{K_{\mathrm{M}}},
$$

and $K_{\mathrm{M}}$ is the Michaelis constant [1]

$$
K_{\mathrm{M}}=\frac{k_{-1}+k_{\mathrm{cat}}}{k_{1}} .
$$

Physically, $\nu$ represents the fraction of the $s=1$ state during one turnover cycle of the enzymatic reaction. It depends only on the substrate concentration and the bare rate constants. In the following analyses, we vary this state parameter $\nu$ to investigate the shear viscosity of enzyme solutions. Some numerical estimates of $\nu$ are given in the end of this section.

We discuss here the validity of using a single-enzyme kinetics. In our model, we have assumed that the concentration of enzymes is small enough so that hydrodynamic interactions between enzymes are negligible [28]. Such a dilute condition corresponds to having only a single enzyme in the system, leading to a renewal process [37]. In the renewal process, the probability distribution function is identically and independently distributed [39]. This means that in every turnover cycle, waiting times follow the same probability distribution, and hence these times can be uniquely determine as shown in Eq. (20).

For systems containing mesoscopic numbers of enzymes, however, stochasticity in enzymatic reactions plays more important roles as discussed in Refs. [39, 40]. Enzyme stochasticity leads to non-renewal processes and causes breakdown of the Michaelis-Menten equation in steady state [39, 40]. Since the waiting time distributions depends on the number of enzymes for non-renewal processes, one needs to derive master equations for waiting time distributions when a solution of multiple enzymes is considered [39]. This is beyond the scope of the present work.

\section{Viscosity of two-state dimer solutions}

To calculate the shear viscosity of a two-state enzyme solution, we introduce the following notations: $\kappa=$ $K_{1} / K_{0}, \lambda=\ell_{1} / \ell_{0}$, and $\lambda^{*}=\ell^{*} / \ell_{0}=(1+\kappa \lambda) /(1+\kappa)$, where $\ell^{*}=\left(K_{0} \ell_{0}+K_{1} \ell_{1}\right) /\left(K_{0}+K_{1}\right)$ is the effective natural length for a dimer in the $s=1$ state. In Appendix C. we show that the viscosity of a two-state enzyme solution is given by

$$
\eta_{\mathrm{e}}(\nu, \epsilon, \kappa, \lambda)=\eta_{0}+\left(\eta_{1}-\eta_{0}\right) \frac{z \nu}{1+z \nu},
$$

where the quantity $\eta_{1}\left(\eta_{0}\right)$ corresponds to the viscosity when all the enzymes are in the $s=1(s=0)$ state

$$
\frac{\eta_{1}(\epsilon, \kappa, \lambda)}{G \tau}=\frac{2 \epsilon}{3} \frac{g_{4}\left(\epsilon(1+\kappa), \lambda^{*}\right)}{g_{2}\left(\epsilon(1+\kappa), \lambda^{*}\right)},
$$

and

$$
z(\epsilon, \kappa, \lambda)=\exp \left[-\frac{\epsilon \kappa}{1+\kappa}(\lambda-1)^{2}\right] \frac{g_{2}\left(\epsilon(1+\kappa), \lambda^{*}\right)}{g_{2}(\epsilon, 1)} .
$$

See also Eq. (11) for the Fraenkel dimer viscosity $\eta_{0}(\epsilon)$. In the above, $g_{m}(p, q)$ is given by an integral

$$
g_{m}(p, q)=\int_{0}^{\infty} d r r^{m} e^{-p(r-q)^{2}}
$$

and its explicit expression is obtained in Appendix C] [see Eq. (C6)]. Specifically, the functions $g_{2}(p, q)(m=2)$ and $g_{4}(p, q)(m=4)$ are given by

$$
g_{2}(p, q)=\frac{q}{2 p} e^{-p q^{2}}+\frac{\sqrt{\pi}\left(1+2 p q^{2}\right)[1+\operatorname{erf}(\sqrt{p} q)]}{4 p^{3 / 2}}
$$




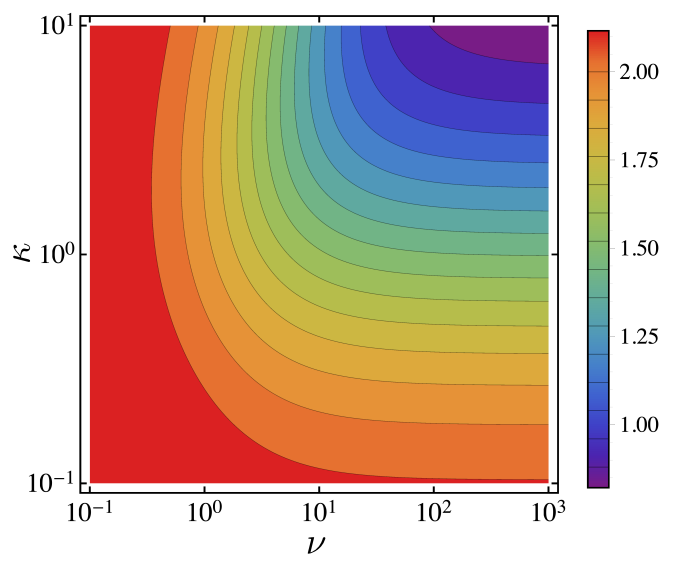

FIG. 3. (Color online) Contour plot of $\eta_{\mathrm{e}} /(G \tau)$ as a function of the parameters $\nu=c_{\mathrm{S}} / K_{\mathrm{M}}$ [see Eq. (22)] and $\kappa=K_{1} / K_{0}$ for $\epsilon=K_{0} \ell_{0}^{2} /\left(2 k_{\mathrm{B}} T\right)=1$ and $\lambda=\ell_{1} / \ell_{0}=1$.

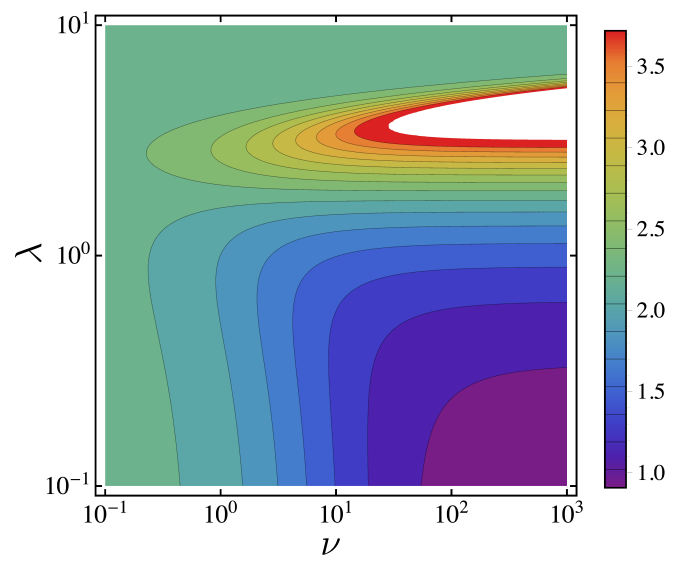

FIG. 4. (Color online) Contour plot of $\eta_{\mathrm{e}} /(G \tau)$ as a function of the parameters $\nu=c_{\mathrm{S}} / K_{\mathrm{M}}$ [see Eq. (22)] and $\lambda=\ell_{1} / \ell_{0}$ for $\epsilon=K_{0} \ell_{0}^{2} /\left(2 k_{\mathrm{B}} T\right)=1$ and $\kappa=K_{1} / K_{0}=1$. The white region corresponds to larger absolute values of $\eta_{\mathrm{e}}$.

and

$$
\begin{aligned}
g_{4}(p, q) & =\frac{q\left(5+2 p q^{2}\right)}{4 p^{2}} e^{-p q^{2}} \\
& +\frac{\sqrt{\pi}\left(3+12 p q^{2}+4 p^{2} q^{4}\right)[1+\operatorname{erf}(\sqrt{p} q)]}{8 p^{5 / 2}},
\end{aligned}
$$

respectively. Equations (24)-(29) for the viscosity are the main result of this work.

In Eq. (26), the factor $\epsilon \kappa /(1+\kappa)(\lambda-1)^{2}$ in the exponential function corresponds to the dimensionless energy difference, $U\left(\ell^{*}, 1\right)-U\left(\ell_{0}, 0\right)$, between the two equilibrium states of a two-state dimer with $\ell_{0}$ and $\ell^{*}$, as shown in Fig. 2(b). Although only the bare reaction rates are taken into account, the above energy difference naturally emerges by defining the weighted distribution function as in Eq. (21).

When $\nu=0, \eta_{\mathrm{e}}$ of Eq. (24) simply reduces to $\eta_{0}$,

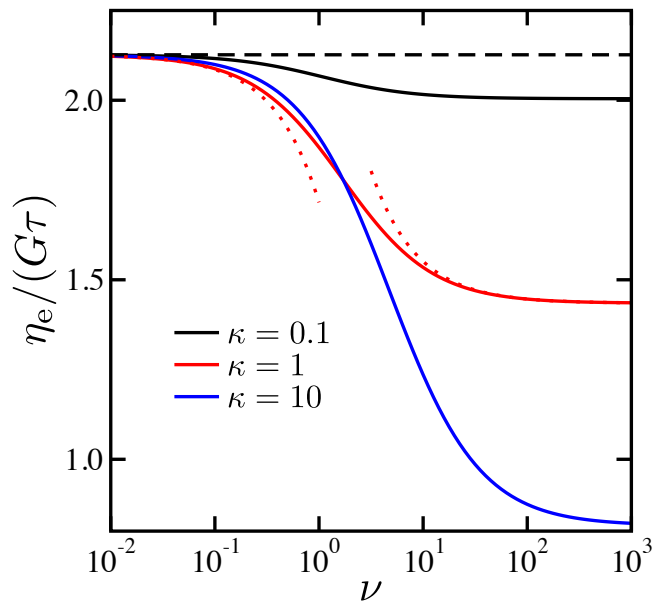

FIG. 5. (Color online) Plot of $\eta_{\mathrm{e}} /(G \tau)$ as a function of the parameter $\nu$ for $\kappa=K_{1} / K_{0}=0.1,1$ and 10. The other parameter values are $\epsilon=K_{0} \ell_{0}^{2} /\left(2 k_{\mathrm{B}} T\right)=1$ and $\lambda=\ell_{1} / \ell_{0}=$ 1. The black dashed line represents $\eta_{0}$ in Eq. (11). The red dotted lines represent the two limiting expressions in Eq. (30) for $\kappa=1$.

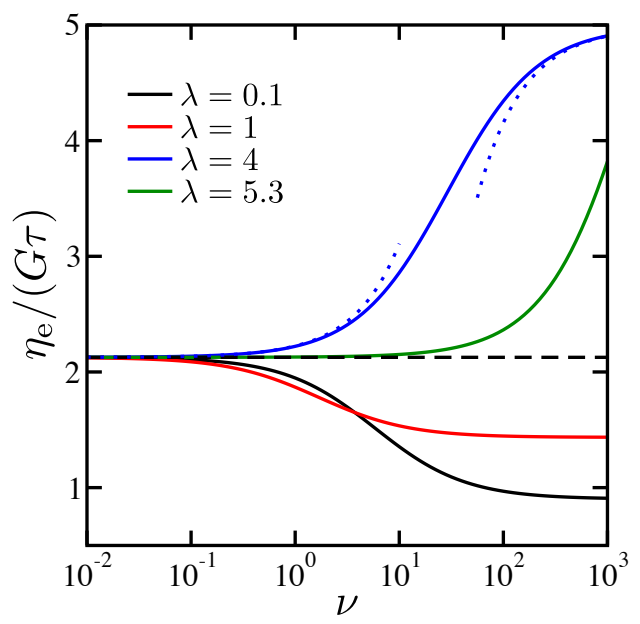

FIG. 6. (Color online) Plot of $\eta_{\mathrm{e}} /(G \tau)$ as a function of the parameter $\nu$ for $\lambda=\ell_{1} / \ell_{0}=0.1,1,4$ and 5.3. The other parameter values are $\epsilon=K_{0} \ell_{0}^{2} /\left(2 k_{\mathrm{B}} T\right)=1$ and $\kappa=K_{1} / K_{0}=$ 1. The black dashed line represents $\eta_{0}$ in Eq. (11). The blue dotted lines represent the two limiting expressions in Eq. (30) for $\lambda=4$.

the viscosity of the Fraenkel dimer solution $[s=0$, see Eq. (11)]. For $\nu \neq 0$, the enzyme solution viscosity $\eta_{\mathrm{e}}$ is determined by the ratio between the two viscosities $\eta_{0}$ and $\eta_{1}$. Due to the factor $z$, however, $\eta_{\mathrm{e}}$ also depends on the energy difference between the two states of the enzyme. This effect causes a non-monotonic behavior of the viscosity as we will show later.

Before analyzing the behavior of $\eta_{\mathrm{e}}$, we estimate typical values of $\epsilon=K_{0} \ell_{0}^{2} /\left(2 k_{\mathrm{B}} T\right)$. The enzymes size can be taken as $\ell_{0} \approx 10 \mathrm{~nm}$ [1]. Moreover, considering typical 
forces, $1 \mathrm{pN}$, generated by a two-state dimer with size $\ell_{0}$, we estimate the spring constant as $K_{0} \approx 10^{-4} \mathrm{~N} / \mathrm{m}[18$. Using these values and $k_{\mathrm{B}} T \approx 4 \times 10^{-21} \mathrm{~J}$ in physiological conditions, we obtain $\epsilon \approx 1$. Hence, we fix the $\epsilon$ value hereafter to $\epsilon=1$.

In Fig. 3, we present the contour plot of the rescaled viscosity due to two-state dimers, $\eta_{\mathrm{e}} /(G \tau)$, as a function of $\nu$ and $\kappa$ for $\epsilon=\lambda=1$. One can see that $\eta_{\mathrm{e}}$ becomes smaller for large $\nu$ and $\kappa$, implying that the viscosity decreases when enzymatic reactions occur more frequently and substrates are stiffer (large $K_{1}$ ). Notice that stiff dimers lead to a decrease of $\eta_{\mathrm{e}}$ because its stiffness suppresses the enzyme size fluctuation. In Fig. 4 , we plot the rescaled viscosity, $\eta_{\mathrm{e}} /(G \tau)$, as a function of $\nu$ and $\lambda$ for $\epsilon=\kappa=1$. Here we see a non-monotonic behavior of the viscosity in $\lambda$ characterized by a peak around $\lambda \approx 3.2$. Note that for larger $\lambda$ values, $\eta_{\mathrm{e}}$ becomes independent of $\nu$.

To see more detailed behavior, we plot in Fig. 5 the rescaled viscosity, $\eta_{\mathrm{e}} /(G \tau)$, as a function of $\nu$ for $\kappa=0.1$, 1 and 10, while keeping $\epsilon=\lambda=1$. The dashed line corresponds to the constant viscosity for a Fraenkel dimer solution, i.e., $\eta_{0} /(G \tau) \approx 2.13$. We see that $\eta_{\mathrm{e}}$ decreases with increasing $\nu$ for all the $\kappa$ values. The decrease of $\eta_{\mathrm{e}}$ is more enhanced for larger $\kappa$ values.

In Fig. 6] we plot $\eta_{\mathrm{e}}$ as a function of $\nu$ for $\lambda=0.1,1,4$ and 5.3, while keeping $\epsilon=\kappa=1$. We see that $\eta_{\mathrm{e}}$ shows both increasing and decreasing dependency as a function of $\nu$ depending on the value of $\lambda$. When $\lambda=0.1,1$, and 4 , the viscosity $\eta_{\mathrm{e}}$ increases with $\lambda$, reflecting the fact that larger enzymes lead to higher viscosity. For larger $\lambda$ such as $\lambda=5.3$, however, $\eta_{\mathrm{e}}$ becomes smaller, and as $\lambda$ is further increased, the viscosity approaches the value of $\eta_{0}$ as indicated by the dashed line. In this limit, both Fraenkel dimer solutions and two-state enzyme solutions exhibit the same viscosity even when $\nu$ is very large.

We discuss now the non-monotonic behavior of $\eta_{\mathrm{e}}$ that is seen in Fig. 6] Such a behavior occurs because $z$ in Eq. (26) increases for smaller $\lambda$, but strongly decreases for larger $\lambda$ due to the Gaussian function of Eq. (26). The factor $\epsilon \kappa(\lambda-1)^{2} /(1+\kappa)$ in the Gaussian function corresponds to the rescaled energy difference between the $s=0$ and $s=1$ states. Hence, it can be regarded as an Arrhenius' equation that determines the transition rate from the $s=0$ to $s=1$ state.

\section{E. Limiting expressions}

Next, we present the limiting expressions of $\eta_{\mathrm{e}}$ for small and large values of the $\nu$ parameter, $\nu \ll 1$ and $\nu \gg$ 1. The viscosity of two-state dimer solution in Eq. (24) becomes

$$
\eta_{\mathrm{e}}(\nu, \epsilon, \kappa, \lambda) \approx \begin{cases}\eta_{0}+C_{1} \nu, & \nu \ll 1 \\ \eta_{1}+\frac{C_{2}}{\nu}, & \nu \gg 1\end{cases}
$$

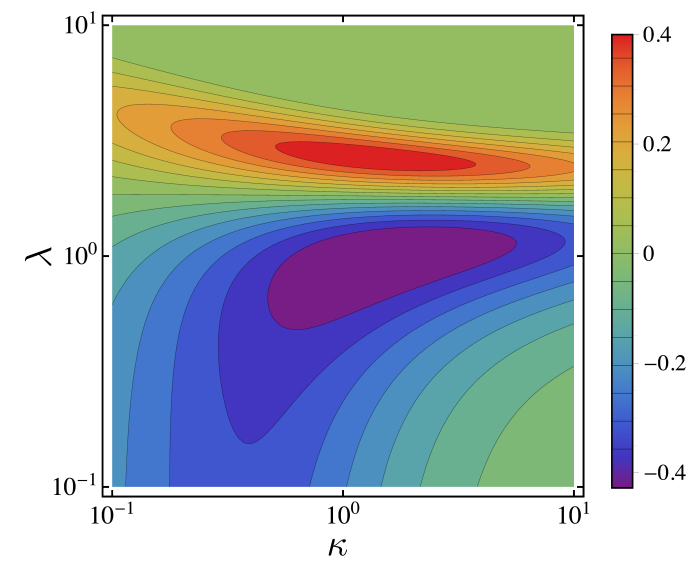

FIG. 7. (Color online) Contour plot of $C_{1} /(G \tau)$ [see Eq. [30)] as a function of $\kappa=K_{1} / K_{0}$ and $\lambda=\ell_{1} / \ell_{0}$ for $\epsilon=K_{0} \ell_{0}^{2} /\left(2 k_{\mathrm{B}} T\right)=1$ under the condition $\nu \ll 1$. The quantity $C_{1}$ changes its sign from negative to positive around $\lambda \approx 2$.

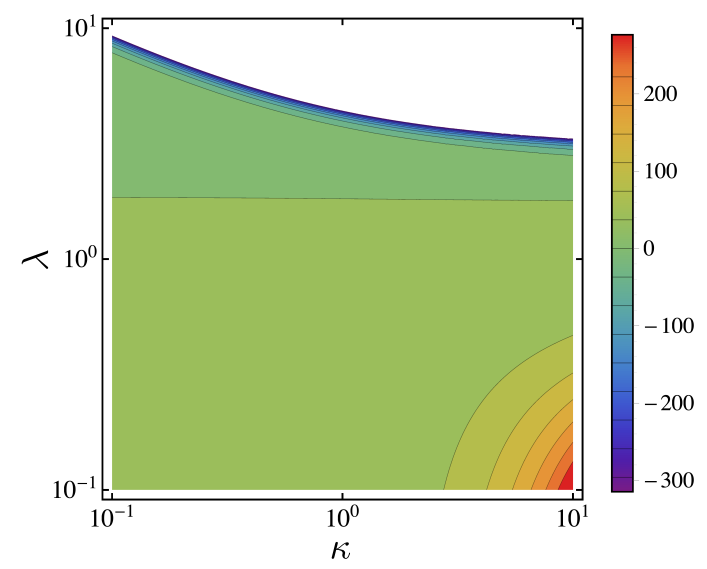

FIG. 8. (Color online) Contour plot of $C_{2} /(G \tau)$ [see Eq. [30] as a function of $\kappa=K_{1} / K_{0}$ and $\lambda=\ell_{1} / \ell_{0}$ for $\epsilon=K_{0} \ell_{0}^{2} /\left(2 k_{\mathrm{B}} T\right)=1$ under the condition $\nu \gg 1$. The quantity $C_{2}$ changes its sign from positive to negative around $\lambda \approx 2$. The white region corresponds to larger absolute values of $C_{2}$.

where $C_{1}(\epsilon, \kappa, \lambda)=\left(\eta_{1}-\eta_{0}\right) z$ and $C_{2}(\epsilon, \kappa, \lambda)=\left(\eta_{0}-\right.$ $\left.\eta_{1}\right) / z$. In Figs. 5and 6 we have plotted the above limits by the red (for $\kappa=1$ ) and blue (for $\lambda=4$ ) dotted line, respectively.

In Fig. 7. we study the $\nu \ll 1$ behavior and plot the coefficient $C_{1}=\left(\eta_{1}-\eta_{0}\right) z$ of $\nu$ in Eq. (30) as a function of $\kappa$ and $\lambda$ for $\epsilon=1$. The behavior of $C_{1}$ is non-monotonic, having a minimum and a maximum around $(\kappa, \lambda) \approx(1,1)$ and $(\kappa, \lambda) \approx(1,2.5)$, respectively. The quantity $C_{1}$ vanishes for large $\lambda$ values, because the Gaussian function in $z$, Eq. (26), dominates over the viscosity difference, $\eta_{0}-\eta_{1}$. Notice that $C_{1}$ changes its sign from negative to positive around $\lambda \approx 2$, where the switching from decreasing to increasing behavior of $\eta_{\mathrm{e}}$ as a function of $\nu$ 
occurs.

In Fig. 8 we study the $\nu \gg 1$ behavior and plot the coefficient $C_{2}=\left(\eta_{0}-\eta_{1}\right) / z$ of $\nu^{-1}$ in Eq. (30) as a function of $\kappa$ and $\lambda$ when $\epsilon=1$. Here $C_{2}$ exhibits a monotonic behavior in $\kappa$ and $\lambda$, and changes its sign from positive to negative around $\lambda \approx 2$. Since $\eta_{\mathrm{e}}$ is inversely proportional to $\nu$ in Eq. (30), positive $C_{2}$ leads to a decreasing behavior of $\eta_{\mathrm{e}}$, whereas negative $C_{2}$ results in an increasing behavior.

\section{F. Numerical estimates}

To end this section, we give some numerical estimates of the parameter $\nu=c_{\mathrm{S}} / K_{\mathrm{M}}$ in Eq. (22). The experimentally accessible substrate concentration is $10^{-6} \mathrm{M}<$ $c_{\mathrm{S}}<10^{-3} \mathrm{M}[12$, 14]. On the other hand, the value of the Michaelis constant $K_{\mathrm{M}}$ differs between fast and slow enzymes. For fast enzymes, such as urease and catalase, it is given by $\left.K_{\mathrm{M}} \approx 10^{-3} \mathrm{M} 99,12\right]$. For slow enzymes, such as aldolase and adenylate kinase, it is $K_{\mathrm{M}} \approx 10^{-6} \mathrm{M}[6,10]$. Hence, the $\nu$ range is estimated as $10^{-3}<\nu<1$ and $1<\nu<10^{3}$, respectively, for fast and slow enzymes. These estimates imply that the limiting expressions derived for $\nu \ll 1$ and $\nu \gg 1$ in Eq. (30) correspond to these two types of enzymes for $c_{\mathrm{S}}<10^{-4} \mathrm{M}$ and $c_{\mathrm{S}}>10^{-5} \mathrm{M}$, respectively.

Next we discuss the values of $\kappa$ and $\lambda$ in order to estimate the viscosity $\eta_{\mathrm{e}}$ for typical physiological conditions. Since an enzyme consists of a large complex of macromolecules, the size of substrate molecules is typically smaller than that of enzymes [1]. Due to this size difference, the condition $\lambda<1$ holds generally. Noncovalent bonds, such as hydrogen bonds, van der Waals attractions and hydrophobic forces, are responsible for the formation of macromolecular assemblies. On the other hand, covalent bonds are responsible for the formation of substrate molecules. Then, the molecular flexibilities for the substrates compared with the enzymes are different, which leads to the condition $\kappa>1$.

From the above argument, we choose $\lambda=0.1$ and $\kappa=10$. Using these values and setting $\epsilon=1$, we obtain $\eta_{\mathrm{e}} /(G \tau) \approx 2.11$ and $\eta_{\mathrm{e}} /(G \tau) \approx 0.39$ for fast and slow enzymes, respectively, assuming that the maximum substrate concentration $c_{\mathrm{S}}=10^{-3} \mathrm{M}$ is attained. Since $\eta_{0} /(G \tau) \approx 2.13$ for $\epsilon=1$, the difference between the enzyme solution with substrates $\eta_{\mathrm{e}}$ and that without substates $\eta_{0}$ is negligible for fast enzymes, whereas the viscosity $\eta_{\mathrm{e}}$ is approximately five times smaller than $\eta_{0}$ for slow enzymes.

\section{DISCUSSION AND CONCLUSION}

In this paper, we have investigated the viscosity of dilute two-state enzyme solutions under steady shear flow. We have obtained the shear viscosity by taking into account the enzyme conformational changes in a solution with a supply of substrates. The waiting times, which correspond to the respective conformations of the enzyme, are connected to the reaction rates in the enzymatic cycle by using the single enzyme kinetics 37]. In our approach, the two-state dimer model [18, 25, 26] and the polymer dimer model [27 29] are combined.

When the enzyme has the same structural properties as the substrate, the shear viscosity decreases as the substrate concentration becomes higher (see Fig. 5). For a substrate larger than the enzyme, the viscosity increases with substrate concentrations (see Fig. 6). When the substrate is large enough, however, the viscosity reduces to that of a Fraenkel dimer solution. Furthermore, we have obtained the limiting expressions of the viscosity for fast and slow enzymes [see Eq. (30)]. For slow enzymes, the coefficient shows only a monotonic behavior. For fast enzymes, on the other hand, the coefficient of the substrate concentration exhibits a non-monotonic behavior as functions of the stiffness and size of the substrate.

Next, we comment on the connection between the viscosity of a two-state dimer solution and the diffusion coefficient of a tracer particle in such a solution. By following the discussion in Refs. [41, 42], the diffusion coefficient of a passive spherical particle of radius $R$ can be given by Einstein's relation

$$
D_{\mathrm{e}}=\frac{k_{\mathrm{B}} T}{6 \pi\left(\eta_{\mathrm{s}}+\eta_{\mathrm{e}}\right) R},
$$

where we have assumed $R \gg \ell_{0}$. In terms of the enzyme volume fraction $\phi=4 \pi\left(\ell_{0} / 2\right)^{3} n / 3, D_{\mathrm{e}}$ can be expanded up to first order in $\phi$ as

$$
D_{\mathrm{e}} \approx \frac{k_{\mathrm{B}} T}{6 \pi \eta_{\mathrm{s}} R}\left(1-\frac{9 a \eta_{\mathrm{e}}}{2 \ell_{0} G \tau} \phi\right) .
$$

Hence, the relative change of the diffusion coefficient with respect to that of a Fraenkel dimer solution (denoted by $\left.D_{0}\right)$ is

$$
\delta D=D_{\mathrm{e}}-D_{0}=\frac{3 k_{\mathrm{B}} T}{4 \pi \eta_{\mathrm{s}} R} \frac{a\left(\eta_{0}-\eta_{\mathrm{e}}\right)}{\ell_{0} G \tau} \phi .
$$

Since $\eta_{0}>\eta_{\mathrm{e}}$ holds for both fast and slow enzymes as estimated before, catalytic enzymes give rise to the diffusion enhancement under physiological conditions. Moreover, we see that $\delta D$ increases as $c_{\mathrm{S}}$ is increased in the limits of fast and slow enzymes (see Figs. 7 and 8). This behavior qualitatively agrees with experiments for both tracers and enzymes [8, 14, 21]. More specifically, using values such as $c_{\mathrm{S}}=10^{-3} \mathrm{M}, a / \ell_{0}=0.2, \phi=0.1$, we obtain that the diffusion increases for slow enzymes as $\delta D / D_{0} \approx 0.15$. In existing experiments, however, $\phi$ is typically of the order of $10^{-5}$, and hence experimental measurements using higher $c_{\mathrm{E}}$ concentration are needed for a more accurately checking of the validity of our model.

Here we discuss how the obtained viscosity is modified by hydrodynamic effects that have been neglected so 
far. In the presence of hydrodynamic interactions, the equation of motion, Eq. (2), can be rewritten as [43]

$$
\frac{\partial r_{\alpha}}{\partial t}=\left(\delta_{\alpha \beta}-\zeta G_{\alpha \beta}\right)\left(\frac{2}{\zeta} f_{\beta}-\frac{2 k_{\mathrm{B}} T}{\zeta} \frac{\partial \ln \psi}{\partial r_{\beta}}\right)+d_{\alpha \beta} r_{\beta},
$$

where $G_{\alpha \beta}(r)=\left(\delta_{\alpha \beta}+r_{\alpha} r_{\beta} / r^{2}\right) /\left(8 \pi \eta_{\mathrm{s}} r\right)$ is the hydrodynamic Oseen tensor [44]. If we assume all orientations to be equally probable, an equilibrium-averaged hydrodynamic interaction can be defined by taking the average of $G_{\alpha \beta}(r)$ over all orientations [45]

$$
h=\frac{1}{3} \operatorname{Tr}\left(\frac{\int d \mathbf{r} \psi(r) G_{\alpha \beta}(r)}{\int d \mathbf{r} \psi(r)}\right),
$$

where $\operatorname{Tr}$ denotes the trace operation. This is called the pre-averaging approximation [44]. Then, the equation of motion can be approximated as

$$
\frac{\partial r_{\alpha}}{\partial t} \approx \frac{2(1-\zeta h)}{\zeta}\left(f_{\alpha}-k_{\mathrm{B}} T \frac{\partial \ln \psi}{\partial r_{\alpha}}\right)+d_{\alpha \beta} r_{\beta} .
$$

Comparing Eqs. (22) and (36), one finds that the change over from negligible hydrodynamic interactions to equilibrium-averaged ones can be accomplished by replacing $\zeta$ with $\zeta /(1-\zeta h)$. Hence, for a single-state dimer as in Eq. (8), the hydrodynamic interaction modifies the viscosity by a factor of $1 /(1-\zeta h)$. In Appendix D we derive $h$ for the Fraenkel dimer model. When $a / \ell_{0}=0.2$ and $\epsilon=1$, for example, we find that the viscosity is about $20 \%$ larger as compared to the negligible hydrodynamic case. For the two-state dimers, hydrodynamic effects do not affect the $\nu$-dependence of $\eta_{\mathrm{e}}$ although some geometrical factors such as $\kappa$ and $\lambda$ can enter in $h$.

In this study, we have assumed that the distribution functions do not depend on shear flow [see Eqs. (10) and [15)]. Here we discuss how these distribution functions are modified by an external flow and the regime where the flow does not affect the distributions as assumed in this paper. For a steady-state homogeneous potential flow, Eq. (5) has an analytical solution 27]

$$
\psi(r)=C^{\prime} \exp \left[-\frac{U(r)}{k_{\mathrm{B}} T}\right] \exp \left[\frac{\zeta}{k_{\mathrm{B}} T} r_{\alpha} d_{\alpha \beta} r_{\beta}\right],
$$

where $C^{\prime}$ is the normalization constant.

For a simple shear flow characterized by a shear rate $\dot{\gamma}$, the distribution function becomes

$\psi(r, \theta, \phi, \dot{\gamma})=C^{\prime} \exp \left[-\frac{U(r)}{k_{\mathrm{B}} T}\right] \exp \left[\frac{\zeta r^{2} \dot{\gamma}}{2 k_{\mathrm{B}} T} \sin ^{2} \theta \sin 2 \phi\right]$,

where $r_{x}=r \sin \theta \cos \phi$ and $r_{y}=r \sin \theta \sin \phi$. When the length of a dimer is $r=\ell_{0}$, the characteristic relaxation time is given by $\zeta \ell_{0}^{2} /\left(k_{\mathrm{B}} T\right)$ [29]. Hence, the shear flow does not affect the distribution functions when $\zeta \ell_{0}^{2} /\left(k_{\mathrm{B}} T\right) \dot{\gamma} \ll 1$.
We have assumed that the transition time spent from one enzymatic species to another is much smaller than the waiting time, i.e., $\tau / W_{s} \ll 1$. Here, we consider the general case of arbitrary waiting time. Because the total times in state $s=0$ and $s=1$ are given by $W_{0}+\tau$ and $W_{1}+\tau_{1}$, respectively, the modified parameter $\nu$ becomes

$$
\nu=\frac{k_{1}\left(1+k_{\mathrm{cat}} \tau_{1}\right) c_{\mathrm{S}}}{k_{-1}+k_{\mathrm{cat}}\left(1+k_{1} \tau c_{\mathrm{S}}\right)},
$$

where $\tau=\zeta /\left(4 K_{0}\right)$ as before and $\tau_{1}=\zeta /\left(4 K_{1}\right)$. Since the reverse reaction rate $k_{-1}$ is negligible in general but may have a finite value, we set it to be a constant. There are only four relevant time scales, namely, $k_{\text {cat }}^{-1},\left(k_{1} c_{\mathrm{S}}\right)^{-1}$, $\tau$, and $\tau_{1}$, and Eq. (39) has four limiting expressions. When the transition rates are vanishingly small, the modified parameter coincides with $\nu$ in Eq. (22) as it should. For the two intermediate regimes, Eq. (39) shows linear and inverse dependences on the transition time. When the transition time is infinitely large, we have $\nu \sim \kappa^{-1}$, indicating that the transition dynamics is governed only by the relative stiffness between the enzyme and substrate.

The transition rates can depend on $\kappa$ and/or $\lambda$ for general enzymatic solutions although these effects were not considered in this work. Using Kramers' reaction-rate theory [46], Aviram et al. [6] obtained free-energy profiles of enzymes by experimentally measuring the transition rates. In the presence of such an effect, the enzyme solution viscosity may exhibit more complicated dependences on $\kappa$ and/or $\lambda$. Finally, we have assumed that the viscosity due to enzymes does not depend on the shear rate. Since the dimer model with finite natural lengths predicts a viscosity that depends on the shear rate [27, 30], one can extend the present model to a non-Newtonian enzymatic fluid.

\section{ACKNOWLEDGMENTS}

We thank R. M. Adar, Y. Avni, K. K. Dey, V. Démery, M. Doi, T. Kato, A. S. Mikhailov, and K. Yasuda for fruitful discussions and helpful suggestions. Y.H. acknowledges support by a Grant-in-Aid for JSPS Fellows (Grant No. 19J20271) from the Japan Society for the Promotion of Science (JSPS). Y.H. also thanks the hospitality of Tel Aviv University, where part of this research was conducted under the TMU-TAU co-tutorial program. S.K. acknowledges the support by Grant-inAid for Scientific Research (C) (Grant No. 18K03567 and No. 19K03765) from the JSPS. D.A. acknowledges support from the Israel Science Foundation (ISF) under grant no. 213/19. 


\section{Appendix A: Probability distribution function for multiple-state enzymes}

In this Appendix, we generalize the dimer-enzyme into a $N$-mer one. We derive the probability distribution function for a single enzyme that has multiple intermediate states in catalytic chemical reactions. We consider the following cascade reaction containing $N$ intermediate substrate-enzyme complexes:

$\mathrm{E}+\mathrm{S} \underset{k_{-1}}{\stackrel{k_{1}}{\rightleftarrows}}(\mathrm{ES})_{1} \underset{k_{-2}}{\stackrel{k_{2}}{\rightleftarrows}} \cdots(\mathrm{ES})_{s} \cdots \underset{k_{-N}}{\stackrel{k_{N}}{\rightleftarrows}}(\mathrm{ES})_{N} \stackrel{k_{\mathrm{cat}}}{\rightleftarrows} \mathrm{E}_{*}+\mathrm{P}$.

Here $(\mathrm{ES})_{s}$ denotes the $s$-th intermediate complex in the reaction, and $k_{s}$ and $k_{-s}$ are the forward and backward reaction rates to the states $s$ and $s-1$, respectively. At the final step, the complex is irreversibly converted to an enzyme and a product with the reaction rate $k_{\text {cat }}$. The enzyme after the catalysis is denoted by $\mathrm{E}_{*}$.

Since we assume that a substrate having the energy $E_{s}$ binds to $(\mathrm{ES})_{s-1}$ with the reaction rate $k_{s}$, the energy of an enzyme in the state $s$ can be written as

$$
U(r, s)=E_{0}+\sum_{s^{\prime}=1}^{s} E_{s^{\prime}},
$$

where $E_{0}$ is the energy of the free enzyme. Then, the waiting time-weighted distribution functions is given by

$$
\psi_{N}(r)=\frac{\sum_{s=0}^{N} W_{s} e^{-\beta U(r, s)}}{\sum_{s=0}^{N} W_{s} \int d \mathbf{r} e^{-\beta U(r, s)}} .
$$

Here $W_{s}$ is the waiting time in the state $s$, which is defined in Eq. (16).

In order to obtain the viscosity of dimer solutions using Eq. (8), we need to calculate the second moment $\left\langle r_{y}^{2}\right\rangle$. In general, the average of any function $f(\mathbf{r})$ over the distribution function, Eq. (A3), can be written as

$$
\begin{aligned}
& \langle f(\mathbf{r})\rangle_{N}=\langle f(\mathbf{r})\rangle_{0} \\
& +\sum_{s=1}^{N}\left[\langle f(\mathbf{r})\rangle_{s}-\langle f(\mathbf{r})\rangle_{0}\right] \frac{z_{s 0} w_{s 0}}{1+\sum_{s^{\prime}=1}^{N} z_{s^{\prime} 0} w_{s^{\prime} 0}},
\end{aligned}
$$

where $\langle f(\mathbf{r})\rangle_{s}$ denotes the average of $f(\mathbf{r})$ over all configurations in the state $s$

$$
\langle f(\mathbf{r})\rangle_{s}=\frac{\int d \mathbf{r} f(\mathbf{r}) e^{-\beta U(r, s)}}{\int d \mathbf{r} e^{-\beta U(r, s)}},
$$

while $z_{s s^{\prime}}$ and $w_{s s^{\prime}}$ are defined by

$$
z_{s s^{\prime}}=\frac{\int d \mathbf{r} e^{-\beta U(r, s)}}{\int d \mathbf{r} e^{-\beta U\left(r, s^{\prime}\right)}}, \quad w_{s s^{\prime}}=\frac{\int_{0}^{\infty} d t p_{s}(t)}{\int_{0}^{\infty} d t p_{s^{\prime}}(t)} .
$$

Notice that the quantity $z$ in Eq. (26) corresponds to $z_{10}$ in the above notation.

\section{Appendix B: Michaelis-Menten kinetics and single enzyme kinetics}

In this Appendix, we briefly review the MichaelisMenten kinetics 34] and the single-enzyme kinetics. In the two-state dimer model, the cascade reaction in Eq. (A1) reduces to the Michaelis-Menten reaction [see Eq. (14)]. In the ensemble of enzymatic experiments, the corresponding kinetic equations become

$$
\begin{aligned}
\frac{d c_{\mathrm{E}}}{d t} & =k_{-1} c_{\mathrm{ES}}-k_{1} c_{\mathrm{E}} c_{\mathrm{S}}, \\
\frac{d c_{\mathrm{ES}}}{d t} & =k_{1} c_{\mathrm{E}} c_{\mathrm{S}}-\left(k_{-1}+k_{\mathrm{cat}}\right) c_{\mathrm{ES}}, \\
\frac{d c_{\mathrm{P}}}{d t} & =k_{\mathrm{cat}} c_{\mathrm{ES}},
\end{aligned}
$$

where $c_{\mathrm{E}}$ and $c_{\mathrm{S}}$ were defined before, whereas $c_{\mathrm{ES}}$ and $c_{\mathrm{P}}$ are the concentrations of substrate-enzyme complex and product, respectively. By replacing the concentrations of the chemical species with the probability distributions, we obtain the kinetic equations for a single enzyme as in Eq. (17). In the steady sate, $d c_{\mathrm{ES}} / d t=0$, the enzymatic velocity is given by

$$
V=\frac{d c_{\mathrm{P}}}{d t}=\frac{V_{\max } c_{\mathrm{S}}}{K_{\mathrm{M}}+c_{\mathrm{S}}},
$$

where $V_{\max }=k_{\text {cat }}\left(c_{\mathrm{E}}+c_{\mathrm{ES}}\right)$ is the maximum enzymatic velocity and $K_{\mathrm{M}}=\left(k_{-1}+k_{\text {cat }}\right) / k_{1}$ is the Michaelis constant defined in Eq. (23).

For a single-enzyme, the corresponding reaction velocity can be obtained from the inverse of the total waiting time during one catalytic cycle. With the use of Eq. (20), this velocity becomes

$$
\frac{1}{W}=\frac{1}{W_{0}+W_{1}}=\frac{k_{\mathrm{cat}} c_{\mathrm{S}}}{K_{\mathrm{M}}+c_{\mathrm{S}}},
$$

which is termed the single-molecule Michaelis-Menten equation [32]. Comparison of Eqs. (B2) and (B3) yields the relation

$$
\frac{V}{c_{\mathrm{E}}+c_{\mathrm{ES}}}=\frac{1}{W} .
$$

This relation originates from the equivalence between the average over a single molecule's long-time trace and that over a large ensemble of identical molecules, i.e., the ergodicity [32, 33].

\section{Appendix C: Derivation of $\eta_{\mathrm{e}}$}

In this Appendix, we present the derivation of $\eta_{\mathrm{e}}$ in Eq. (24). Using Eq. (21), we calculate $\left\langle r_{y}^{2}\right\rangle$ in Eq. (8) as

$$
\eta_{\mathrm{e}}=\frac{n \zeta}{4} \frac{\int d \mathbf{r}\left[r_{y}^{2} e^{-\beta U(r, 0)}+\nu r_{y}^{2} e^{-\beta U(r, 1)}\right]}{\int d \mathbf{r}\left[e^{-\beta U(r, 0)}+\nu e^{-\beta U(r, 1)}\right]}
$$


With the use of Eq. for $N=1$, we obtain

$$
\eta_{\mathrm{e}}=\frac{n \zeta}{4}\left(\left\langle r_{y}^{2}\right\rangle_{0}+\left[\left\langle r_{y}^{2}\right\rangle_{1}-\left\langle r_{y}^{2}\right\rangle_{0}\right] \frac{z \nu}{1+z \nu}\right) .
$$

Since $n \zeta\left\langle r_{y}^{2}\right\rangle_{0} / 4=\eta_{0}$ and $n \zeta\left\langle r_{y}^{2}\right\rangle_{1} / 4=\eta_{1}$, we obtain Eq. (24). The viscosity of a Fraenkel dimer solution $\eta_{0}$ is given by Eq. (11).

Next we calculate $\eta_{1}$ in Eq. (25) as

$$
\eta_{1}=\frac{n \zeta}{4} \frac{\int d \mathbf{r} r_{y}^{2} e^{-\beta U(r, 1)}}{\int d \mathbf{r} e^{-\beta U(r, 1)}}=\frac{n \zeta}{12} \frac{\int_{0}^{\infty} d r r^{4} e^{-\beta U(r, 1)}}{\int_{0}^{\infty} d r r^{2} e^{-\beta U(r, 1)}}
$$

For a harmonic potential, the integration of $r^{m}$ can be generally expressed as

$$
\begin{aligned}
g_{m}(p, q) & =\int_{0}^{\infty} d r r^{m} e^{-p(r-q)^{2}} \\
& =\int_{-q}^{\infty} d u(u+q)^{m} e^{-p u^{2}} \\
& =\sum_{n=0}^{m} \frac{m !}{(m-n) ! n !} q^{m-n} \int_{-q}^{\infty} d u u^{n} e^{-p u^{2}} .
\end{aligned}
$$

The last integral can be further performed as follows.

$$
\begin{aligned}
& \int_{-q}^{0} d u u^{n} e^{-p u^{2}}+\int_{0}^{\infty} d u u^{n} e^{-p u^{2}} \\
& =\frac{p^{-(n+1) / 2}}{2}\left[(-1)^{n} \int_{0}^{p q^{2}} d t t^{(n+1) / 2-1} e^{-t}\right. \\
& \left.+\int_{0}^{\infty} d t t^{(n+1) / 2-1} e^{-t}\right] \\
& =\frac{p^{-(n+1) / 2}}{2}\left[\left[1+(-1)^{n}\right] \int_{0}^{\infty} d t t^{(n+1) / 2-1} e^{-t}\right. \\
& \left.-(-1)^{n} \int_{p q^{2}}^{\infty} d t t^{(n+1) / 2-1} e^{-t}\right]
\end{aligned}
$$

Finally, $g_{m}(p, q)$ becomes

$$
\begin{aligned}
& g_{m}(p, q)=\frac{1}{2} \sum_{n=0}^{m} \frac{m !}{(m-n) ! n !} p^{-(n+1) / 2} q^{m-n} \\
& \times\left[\left[1+(-1)^{n}\right] \Gamma\left(\frac{n+1}{2}\right)-(-1)^{n} \Gamma\left(\frac{n+1}{2}, p q^{2}\right)\right],
\end{aligned}
$$

where $\Gamma(x)=\int_{0}^{\infty} d t t^{x-1} e^{-t}$ and $\Gamma(x, \alpha)=$ $\int_{\alpha}^{\infty} d t t^{x-1} e^{-t}$ are the gamma function and the incomplete gamma function of the second kind, respectively [31].

\section{Appendix D: Hydrodynamic interactions between two spheres}

In this Appendix, we present the calculation of Eq. 35 for the Fraenkel dimer model. With the assumption that the fluid is isotropic, the Oseen tensor becomes $\delta_{\alpha \beta} /\left(6 \pi \eta_{\mathrm{s}} r\right)$. Substituting it into Eq. (35) yields

$$
h=\frac{1}{6 \pi \eta_{\mathrm{s}}} \frac{\int d \mathbf{r} \psi_{0}(r) / r}{\int d \mathbf{r} \psi_{0}(r)}
$$

By taking $m=1,2$ in $g_{m}(p, q)$, Eq. (C6), the dimensionless combination $\zeta h$ is obtained as

$$
\begin{aligned}
\zeta h(\epsilon) & =\frac{a}{\ell_{0}} \frac{g_{1}(\epsilon, 1)}{g_{2}(\epsilon, 1)} \\
& =\frac{a}{\ell_{0}} \frac{e^{-\epsilon}+\sqrt{\pi \epsilon}[1+\operatorname{erf}(\sqrt{\epsilon})]}{e^{-\epsilon}+\sqrt{\pi \epsilon}[1+1 /(2 \epsilon)][1+\operatorname{erf}(\sqrt{\epsilon})]} .
\end{aligned}
$$

For large dimers, $a / \ell_{0} \ll 1$, the hydrodynamic effects become negligible. The limiting behavior of $h$ for the Hookean, $\epsilon \ll 1$, and stiff Fraenkel dimers, $\epsilon \gg 1$, is given, respectively, by

$$
\zeta h(\epsilon)= \begin{cases}\frac{2 a}{\ell_{0}} \sqrt{\frac{\epsilon}{\pi}} & \epsilon \ll 1, \\ \frac{a}{\ell_{0}} \frac{1}{1+1 /(2 \epsilon)} & \epsilon \gg 1 .\end{cases}
$$

[1] B. Alberts, A. Johnson, P. Walter, J. Lewis, and M. Raff, Molecular Biology of the Cell (Garland Science, New York, 2008).

[2] M. Gerstein, A. M. Lesk, and C. Chothia, Biochemistry 33, 6739 (1994).

[3] Y. Togashi and A. S. Mikhailov, Proc. Natl. Acad. Sci. (USA) 104, 8697 (2007).

[4] T. Sakaue, R. Kapral, and A. S. Mikhailov, Eur. Phys. J. B 75, 381 (2010).
[5] C. Echeverria, Y. Togashi, A. S. Mikhailov, and R. Kapral, Phys. Chem. Chem. Phys. 13, 10527 (2011).

[6] H. Y. Aviram, M. Pirchi, H. Mazal, Y. Barak, I. Riven, and G. Haran, Proc. Natl. Acad. Sci. (USA) 115, 3243 (2018).

[7] Y. Zhang and H. Hess, ACS Cent. Sci. 5, 939 (2019).

[8] H. S. Muddana, S. Sengupta, T. E. Mallouk, A. Sen, and P. J. Butler, J. Am. Chem. Soc. 132, 2110 (2010).

[9] C. Riedel, R. Gabizon, C. A. M. Wilson, K. Hamadani, 
K. Tsekouras, S. Marqusee, S. Pressé, and C. Bustamante, Nature 517, 227 (2015).

[10] P. Illien, X. Zhao, K. K. Dey, P. J. Butler, A. Sen, and R. Golestanian, Nano Lett. 17, 4415 (2017).

[11] S. Sengupta, K. K. Dey, H. S. Muddana, T. Tabouillot, M. E. Ibele, P. J. Butler, and A. Sen, J. Am. Chem. Soc. 135, 1406 (2013).

[12] A.-Y. Jee, S. Dutta, Y.-K. Cho, T. Tlusty, and S. Granick, Proc. Natl. Acad. Sci. (USA) 115, 14 (2018).

[13] K. K. Dey, F. Y. Pong, J. Breffke, R. Pavlick, E. Hatzakis, C. Pacheco, and A. Sen, Angew. Chem. Int. Ed. 55, 1113 (2016).

[14] X. Zhao, K. K. Dey, S. Jeganathan, P. J. Butler, U. M. Córdova-Figueroa, and A. Sen, Nano Lett. 17, 4807 (2017).

[15] P. Illien, T. Adeleke-Larodo, and R. Golestanian, EPL 119, 40002 (2017).

[16] T. Adeleke-Larodo, P. Illien, and R. Golestanian, Eur. Phys. J. E 42, 39 (2019).

[17] R. Golestanian, Phys. Rev. Lett. 115, 108102 (2015).

[18] A. S. Mikhailov and R. Kapral, Proc. Natl. Acad. Sci. (USA) 112, E3639 (2015).

[19] R. Kapral and A. S. Mikhailov, Physica D 318-319, 100 (2016).

[20] Y. Hosaka, K. Yasuda, R. Okamoto, and S. Komura, Phys. Rev. E 95, 052407 (2017).

[21] M. Xu, J. L. Ross, L. Valdez, and A. Sen, Phys. Rev. Lett. 123, 128101 (2019).

[22] Y. Zhang, M. J. Armstrong, N. M. B. Kazeruni, and H. Hess, Nano Lett. 18, 8025 (2018).

[23] J.-P. Günther, G. Majer, and P. Fischer, J. Chem. Phys. 150, 124201 (2019).

[24] V. Armoškaitè, K. Ramanauskienè, and V. Briedis, Afr. J. Pham. Pharmacol. 6, 1685 (2012).

[25] H. Flechsig and A. S. Mikhailov, J. R. Soc. Interface 16, 20190244 (2019).

[26] Y. Hosaka, S. Komura, and A. S. Mikhailov, unpublished.
[27] R. B. Bird, R. C. Armstrong, O. Hassager, and C. F. Curtiss, Dynamics of Polymeric Liquids, Vol. 2 (Wiley, New York, 1987).

[28] M. Doi, Soft Matter Physics (Oxford University, Oxford, 2013).

[29] G. K. Fraenkel, J. Chem. Phys. 20, 642 (1952).

[30] R. B. Bird, C. F. Curtiss, and K. J. Beers, Rheol. Acta. 36, 269 (1997).

[31] M. Abramowitz and I.A. Stegun, Handbook of Mathematical Functions (Dover, New York, 1972).

[32] S. C. Kou, B. J. Cherayil, W. Min, B. P. English, and X. S. Xie, J. Phys. Chem. 109, 19068 (2005).

[33] B. P. English, W. Min, A. M. van Oijen, K. T. Lee, G. Luo, H. Sun, B. J. Cherayil, S. C. Kou, and X. S. Xie, Nature Chem. Bio. 2, 87 (2006).

[34] L. Michaelis and M. L. Menten, Biochem. Z. 49, 333-369 (1913).

[35] J. Cao, J. Phys. Chem. B 115, 5493 (2011).

[36] N. G. van Kampen, Stochastic processes in physics and chemistry (Elsevier Science, New York, 1992).

[37] H. P. Lu, L. Xun, and X. S. Xie, Science 282, 1877 (1998).

[38] S. Xie, Single Mol. 2, 229 (2001).

[39] S. Saha, S. Ghose, R. Adhikari, and A. Dua, Phys. Rev. Lett. 107, 218301 (2011).

[40] R. Grima, Phys. Rev. Lett. 102, 218103 (2009).

[41] N. Oppenheimer and H. Diamant, Biophys. J. 96, 3041 (2009).

[42] N. Oppenheimer and H. Diamant, Phys. Rev. E 82, 041912 (2010).

[43] R. B. Bird and H. R. Warner, Trans. Soc. Rheol. 15, 741 (1971).

[44] M. Doi and S. F. Edwards, The Theory of Polymer Dynamics (Oxford University, New York, 1986).

[45] H. R. Warner, Ind. Eng. Chem. Fundam., 11, 379 (1972).

[46] P. Hänggi, P. Talkner, and M. Brokovec, Rev. Mod. Phys. 62, 251 (1990). 